\title{
Marker-assisted breeding for Downy mildew, Powderey mildew and Phylloxera resistance at FEM
}

\author{
Silvia Vezzulli, ${ }^{1,}{ }^{*}$, Chiara Dolzani ${ }^{1}$, Daniela Nicolini $^{1}$, Paola Bettinelli ${ }^{1}$, Daniele Migliaro ${ }^{2}$, Verena Gratl $^{3}$, \\ Tommaso Stedile ${ }^{l}$, Alessandra Zatelli ${ }^{1}$, Monica Dallaserra ${ }^{l}$, Silvano Clementi ${ }^{1}$, Cinzia Dorigatti ${ }^{1}$, Riccardo \\ Velasco $^{1}$, Luca Zulini $^{1}$, and Marco Stefanini ${ }^{l}$ \\ ${ }^{1}$ Research and Innovation Centre, Fondazione Edmund Mach, Via E. Mach 1, 38010 San Michele all'Adige, Italy \\ ${ }^{2}$ Consiglio per la Ricerca in Agricoltura e l'analisi dell'economia agraria - Centro di Ricerca per la Viticoltura (CREA- \\ VIT), viale XXVIII Aprile 26, I-31015 Conegliano (TV) \\ ${ }^{3}$ Centrum of Chemistry and Biomedicine, University of Innsbruck, Innrain 80/82, A-6020 Innsbruck
}

\begin{abstract}
Il programma di miglioramento genetico per le resistenze a stress biotici ha avuto inizio presso la Fondazione Edmund Mach (FEM) nel 2010. Inizialmente è stata condotta una caratterizzazione sia genotipica che fenotipica di materiali acquisiti da altri programmi di breeding e di materiale selvatico raccolto in New Jersey. Sia i genotipi conosciuti nei database internazionali che i genotipi sconosciuti, imparentati e non, sono stati impiegati come linee parentali nel processo di introgressione e di piramidazione di loci di interesse. Una volta pianificati e ottenuti gli incroci, la valutazione delle progenie è avvenuta seguendo un processo di Marker-Assisted Selection: dapprima è avvenuta la selezione fenotipica in serra in base al tipo di malattia e al numero di loci attesi per la medesima malattia; successivamente si è proceduto con lo screening molecolare in base ai loci specifici attesi nei parentali. Cinque sono i loci Run/Ren associati alla resistenza all'oidio presenti nel programma FEM; riguardo ai loci associati alla resistenza alla peronospora, quattro Rpv sono ben rappresentati nel piano di incroci. Ad oggi il 26\% delle F1 è piramidizzato per quattro loci di resistenza.
\end{abstract}

\begin{abstract}
The genetic improvement program for resistance to biotic stresses began at the Edmund Mach Foundation (FEM) in 2010. Initially, both genotypic and phenotypic characterization of materials acquired from other breeding programs and wild material collected in New Jersey was conducted. Both the genotypes known in the international databases and the unknown genotypes, both related and unrelated, were used as parental lines in the process of introgression and pyramiding of loci of interest. Once the crossbreeds were planned and obtained, the evaluation of the progeny took place following a Marker-Assisted Selection process: at first the phenotypic selection in the greenhouse occurred based on the type of disease and the number of loci expected for the same disease; then we proceeded with molecular screening based on the specific loci expected in the parental groups. Five Run/Ren loci are associated with the resistance to powdery mildew present in the FEM program; with respect to the loci associated with the downy mildew resistance, four $R p v$ loci are well represented in the crossing plan. To date, $26 \%$ of $\mathrm{F} 1$ is pyramided for four resistance loci.
\end{abstract}

\section{Introduction}

Downy mildew (DM) and powdery mildew (PM) are the two most important plagues affecting viticulture. The first disease is caused by the oomycete Plasmopara viticola (Berl. \& De Toni), while the second one by the ascomycete Erysiphe necator (Schwein.). Both reduce fruit quality and yield, either by direct infection of berries or as a negative result caused by leaf infections $[1,2]$. Their control is based on the massive use of fungicides, leading to problems such as environmental pollution and pathogen resistance development. The use of grapevine varieties showing durable resistance to DM and PM is a promising strategy. Unlike in almost all of Vitis vinifera varieties, sources of genetic resistance against $P$. viticola and $E$. necator were identified in a range of American and Asian wild Vitis species and used in inter-specific breeding programs through backcrossing to obtain resistant genotypes with acceptable quality features and considerable mildew resistance

* Corresponding author: silvia.vezzulli@,fmach.it 
characteristics [3]. In an Old World country such as Italy, the demand for innovation has historically been addressed to increase the complexity of wines. However, during the last decade the need for new varieties with vinifera-like qualitative content and (mid-)resistance to pathogens has emerged among grapevine growers, nursery workers, and end-users. In order to accelerate the process of selection of resistant genotypes, one must consider genetics and genomics. In particular, given parent lines of the donors of resistance sources, the Marker-Assisted Selection (MAS) approach is based on the tracking of resistance loci $(R$-loci) that contain genes associated with the resistance to pathogens [4].

The program of genetic improvement for resistance to biotic stresses, which began at the Edmund Mach Foundation (FEM) in 2010, has two objectives: the first is to develop new pathogen resistant varieties, with a high level of quality, that are ready for placing on the market, while the second is to develop resistance superdonors that then act as more efficient parents for future breeding programs, releasing the resistance trait(s) to all the progeny. Hereafter, the second FEM optimized Marker-Assisted Breeding (MAB) strategy is described.

\section{Materials and methods}

\subsection{Genetic material}

Parental lines. A total of 264 grapevine accessions were studied. They included 220 non-vinifera accessions acquired from (non-)European breeding programs, an Italian private breeding platform, wild-collected samples in north-eastern America during 2011, and $44 \mathrm{~V}$. vinifera varieties. Most were phenotyped for DM and PM resistance, while all were genetically characterized. Progenies. During the last 6 years a total of 86 different crossing combinations $(V$. hybrid $\times V$. hybrid) were performed aiming at pyramiding $R$-loci. A total of 8793 progeny individuals were submitted to the MAS workflow, namely first by disease resistance screening and second by $R$-loci characterization.

\subsection{Phenotypic screening}

Parental lines. Within the experimental genetic material, 100 accessions physically available at FEM were evaluated for their degree of resistance against DM and $\mathrm{PM}$, through in vitro leaf disc bioassays and in vivo pathogen inoculation on potted plants respectively. DM symptom annotation was performed based on three parameters: Severity (percentage of the disc area showing symptoms of sporulation), Incidence (number of discs with sporulation/total number of discs), according to OEPP/EPPO [5], and the OIV 452-1 descriptor [8]. PM symptoms were evaluated based on the foliar OIV 455 descriptor. Progenies. All progeny individuals were evaluated for their degree of resistance against downy and powdery mildew. Firstly, $P$. viticola spores were inoculated on potted seedlings following a well-established protocol [6]; secondly, prior to plant treatment and recovery, $E$. necator artificial infection was caused by naturally infected plants adjacent to the potted seedlings. The assessments were recorded respectively at 7 and 14 days post-inoculation (dpi) based on the OIV 452 and 455 descriptors.

\subsection{Genotypic characterization}

Parental lines. Following a detailed literature search, 11 reliable $R$-loci were analyzed in the entire parental sample set: i) 6 associated to PM resistance, Run1, Run2, $\operatorname{Ren} 1, \operatorname{Ren} 2, \operatorname{Ren} 3, \operatorname{Ren} 9$; ii) 5 associated to DM resistance, Rpv1, Rpv3, Rpv10, Rpv12, Rpv14. The applied PCR conditions were those reported in the overall literature, with some modifications [9]. Progenies. The progeny individuals maintained downstream of the phenotypic screening which performed well were finally characterized at the expected $R$-loci based on the parent combinations.

\section{Results and discussion}

Parental lines. Within the 100 physically available accessions, the in vitro evaluation demonstrated a wide phenotypic variability in terms of DM resistance. In particular, 41 accessions resulted in highly resistant material (OIV scores 7-9) and will be employed as a direct source of resistance in breeding sub-programs with the objective of releasing novel sustainable grapevine cultivars presenting good fruit/wine quality (e.g. good yield, absence of off-flavours). Regarding $\mathrm{PM}$, only 18 out of the 100 in vivo tested accessions resulted in resistant materials. This demonstrates that PM resistance is rarer than DM resistance and highlights the importance of discovering new genetic sources. Among these accessions, a deep phenotypic characterization has recently been reported for 28 resistant varieties [7].

The following genetic study allowed the identification of already pyramided parents obtained in the past by traditional breeding activities. Moreover, most genotypes were found with no presence of any of the 11 reliable and actually analysable $R$-loci, representing potentially novel and exclusive genetic resources (Fig. 1). 
A.

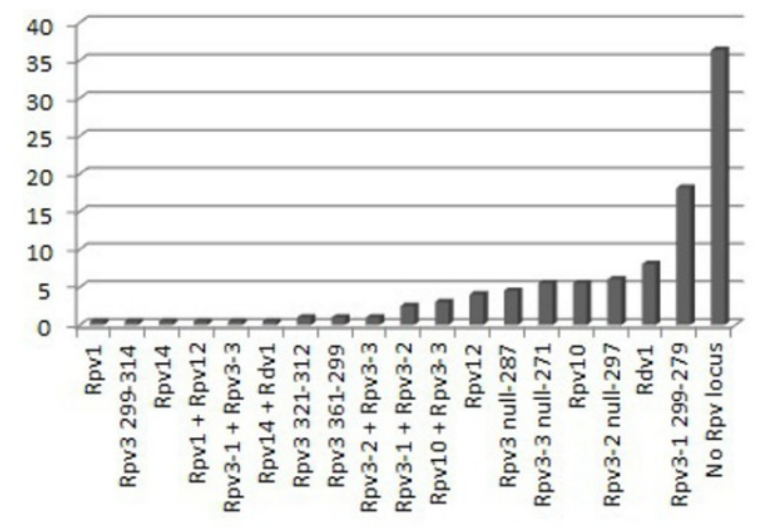

B.

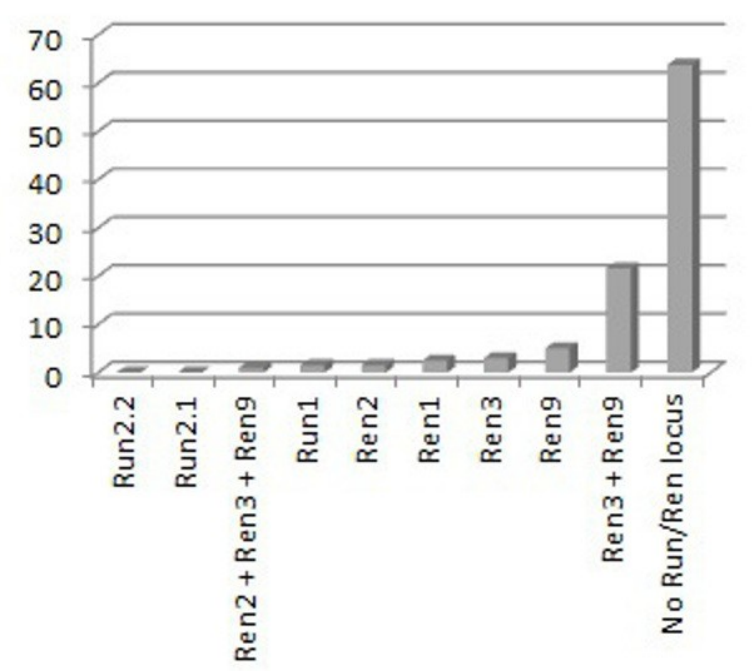

Fig. 1. Rpv (A) and Run/Ren (B) loci distribution in the overall parental genetic material.

Progenies. Out of the total 8793 plants subjected to DM and PM resistance screening, 6182 were eliminate due to their high susceptibility level to both disease while 2611 $(29.7 \%)$ were maintained in order to perform the $R$-loci characterization. Table 1 shows the specific classes, based on the OIV scores (1-9), into which the progeny individuals maintainedwere classified.

Tab. 1. Efficiency of the phenotypic screening for DM and PM resistance in progenies derived from $V$. hybrid $\times V$. hybrid during 2013, 2014, 2015 and 2017.

\begin{tabular}{|c|c|c|}
\hline Total of inoculated plants & 8793 & $100 \%$ \\
\cline { 1 - 2 } DM 9 PM 9 & 623 & $7.1 \%$ \\
\cline { 1 - 1 } DM 9 PM 7-9 & 3171 & $20.1 \%$ \\
\cline { 1 - 1 } DM 9 PM 5 & $0.4 \%$ \\
\cline { 1 - 2 } DM 5-7 PM 9 & 14 & $0.5 \%$ \\
\cline { 1 - 1 } DM 5-7 PM 7 & 123 & $1.4 \%$ \\
\cline { 1 - 1 } DM 5-7 PM 5 & $6.2 \%$ \\
\cline { 1 - 1 } Total of discarded plants DM 1-3 PM 1-3 & 6182 & $70.3 \%$ \\
\cline { 1 - 1 } Total of maintained plants & 2611 & $29.7 \%$ \\
\hline
\end{tabular}

Out of the 2611 progeny individuals retained subsequent to the phenotypic screening, 963 plants showing the highest degree of resistance and the best agronomic performance were subjected to the genotyping analysis in order to define both the number and the typology of $R$-loci. Besides $11.1 \%$ of genotypes carrying a single $R$ locus associated to DM or PM resistance, our findings highlighted the pyramidization of $R$-loci against DM in $48.4 \%$ and against PM in $59.5 \%$ of the analysed progeny individuals. In particular, $30.3 \%$ of genotypes showed to be pyramided for $R$-loci to both mildews. Overall, the most abundant class (30.9\%) was represented by genotypes carrying $3 R$-loci, while a maximum level of pyramiding with $7 R$-loci was reached in $0.3 \%$ of cases (Fig. 2).

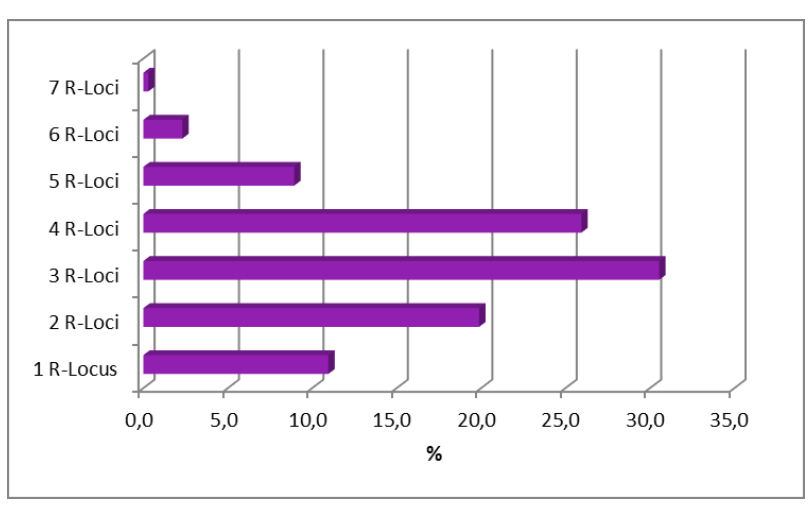

Fig. 2. Level of $R$-loci pyramiding in the analyzed progeny individuals.

\section{Conclusions}

The present survey allowed the identification of already pyramided parents obtained in the past by traditional breeding activities; this supports the role that genetics, coupled with genomics, plays in assisting genetic improvement for resistance. Moreover, novel and exclusive genetic resources were identified, providing peculiar and preparatory information for ongoing and forthcoming Marker-Assisted (pre-)Breeding programs. Following a MAS workflow, nowadays a relevant level of $R p v$ and Run/Ren loci pyramiding has been reached in the obtained selections. In the next coming few years, FEM is going to launch eight new varieties - carrying from 1 to $3 R$-loci - which are at least mid-resistant to $\mathrm{DM}$ and PM.

The authors are grateful to Erhard Tutzer (Innovitis, IT) for providing already known and recently developed grapevine genotypes. The authors also thank M. Stella Grando and S. Lorenzi for their initial contribution to the work.

\section{References}

1. C. Gessler, I. Pertot, M. Perazzolli, Phytopathol. Mediterr., 50, 3-44 (2011) 
2. M.A. Ellis. Agriculture and Natural Resources, 1-3 (2008)

3. R. Eibach, R. Töpfer. Grapevine breeding programs for the wine industry (Woodhead Publishing, 3-4, 2015)

4. B.C.Y. Collard, D.J. Mackill, Phil. Trans. R. Soc. B., 363, 557-572 (2008)

5. OEPP/EPPO. Guidelines for the efficacy evaluation of fungicides, OЕPР/EPPO Bullettin, 31, 313-317 (2001)

6. D. Buonassisi, L. Cappellin, C. Dolzani, R. Velasco, E. Peressotti, S. Vezzulli, Sci. Hort., 236, 79-89 (2018)

7. S. Vezzulli, A. Vecchione, M. Stefanini, L. Zulini, Eur. J. Plant Pathol., 150, 485-495 (2018)

\section{Web references}

8. OIV 2009. Descriptor list for grape varieties and Vitis species, $2^{\text {nd }}$ edn. Office International de la Vigne et du Vin, Paris, http://www.oiv.org

9. VIVC 2018. Vitis International Variety Catalogue. http://www.vivc.de/index.php 